\title{
Embryonic Inner Ear Cells Reaggregate into Specific Patterns In Vitro
}

\author{
Lynne M. Bianchi, Abigail L. Person, and Ellen B. Penney \\ Neuroscience Program, Oberlin College, Oberlin, OH 44074, USA
}

Received: 28 August 2001; Accepted: 14 January 2002; Online publication: 26 March 2002

\begin{abstract}
The sensory epithelia of the mammalian inner ear consist of a highly precise pattern of sensory hair cells and supporting cells. The mechanisms regulating this patterning are only beginning to be determined. The present study describes a method for culturing dissociated embryonic inner ear cells and the resulting patterning that occurs in these cultures. The results indicate that developing inner ear cells aggregate into precise patterns on a two-dimensional substrate, suggesting that intrinsic patterning mechanisms remain active in vitro. Using antibodies and scanning electron microscopy to detect hair cells and nonsensory cells, it was determined that only a subset of aggregates contained sensory hair cells. The hair cells were organized into specific patterns and surrounded by supporting cells, similar to the in vivo pattern. Additionally, hair cells increased their immunoreactivity and number of stereocilia over time, suggesting that hair cells continue to mature in vitro. Thus, the study reveals that the cells of the developing inner ear provide the necessary signals that direct sensory hair cells and supporting cells to reassociate into very precise patterns in vitro and that these patterns are reminiscent of the patterning that occurs in vivo.
\end{abstract}

Keywords: hair cell development, cochlea, auditory, vestibular, otocyst, calretinin, myosin VIIa

Correspondence to: Dr. Lynne M. Bianchi • Neuroscience Program • Oberlin College • 130 West Lorain Street. - Oberlin, OH 44074. Telephone: (440) 775-8369; fax: (440) 775-8960;

email: lynne.bianchi@oberlin.edu

\section{INTRODUCTION}

The inner ear arises from the otocyst, a simple, fluidfilled sac that gives rise to all of the sensory epithelial regions of the inner ear as well as the statoacoustic ganglion. The epithelial regions of the inner ear are arranged in a precise pattern of supporting cells and sensory hair cells. The cells are arranged so that hair cells normally contact adjacent supporting cells but not one another (reviewed by Kelley and Bianchi 2001). How the various cell types that originate from the otocyst ultimately organize into the characteristic cellular mosaic of the sensory epithelium is unclear. The epithelium itself appears to contain the signals necessary for such patterning. Pioneering studies of Orr (1968) demonstrated that dissociated chick otocyst cells would reaggregate into specific cell groups when placed in roller cultures for 3-12 days. The cells aggregated into clumps of epithelial-lined structures, connective tissue, and ganglion cells during the culture period. The observation that the dissociated cells aggregated and continued to develop into recognizable structures supports the hypothesis that inner ear cell patterns are established through specific molecules. Due to the lack of available markers at that time, the precise cellular constituents of the aggregates could not be determined.

More recently, markers for hair cells and nonsensory cells have been identified. Hair cell markers include anti-calretinin, anti-myosin VIIa, and anti-pv3 antibodies. Calretinin is a calcium-binding protein that is expressed in mouse vestibular hair cells beginning at E17 and in cochlear inner hair cells beginning at E19 (Dechesne et al. 1994). Myosin VIIa, an unconventional molecular motor, is expressed in mouse vestibular hair cells beginning at E14.5 and in cochlear hair cells after E16.5 (Hasson et al. 1997; 
Self et al. 1998). A third hair cell marker, parvalbumin 3 (pv3), is a calcium-binding protein that is restricted to hair cells and is detected as soon as hair cells differentiate in bullfrog, zebra fish, chick, and mouse (Heller 2001). Few markers to identify specific supporting cells have been reported; however, anticytokeratin antibodies have been used to identify nonsensory cells in the inner ear (Flock et al. 1982; Kasper et al. 1987; Stone et al. 1996; Lawlor et al. 1999). The availability of such markers now permits the identification of cells in vitro.

In the present study, an in vitro assay was developed to test whether cell aggregation and patterning also occurred in mammalian inner ear cells. Inner ear tissues were harvested from embryonic mice at days 12-14, periods of active development of the mouse inner ear. E12 is a similar stage of development to that used in the chick studies (Orr 1968) and is a period prior to hair cell differentiation in vivo. By E14, some, but not all, hair cells have committed to the hair cell fate (reviewed by Kelley and Bianchi 2001). The entire inner ear was used to see if sensory and nonsensory cells from the cochlear and vestibular regions would sort out into specific cellular patterns when placed in random order. The markers for hair cells and supporting cells, as well as scanning electron microscopy (SEM), were used to investigate how specific inner ear cell types associated in the two-dimensional cultures. The results revealed that mammalian inner ear cells aggregated into specific patterns in vitro and only a subset of aggregates contained hair cells. Hair cells were adjacent to supporting cells, similar to the in vivo pattern. Additionally, the time course for hair cell immunoreactivity was similar to that observed in vivo and stereocilia bundles increased in number over time. These results suggest inner ear cells grown in vitro use intrinsic patterning and maturational cues similar to those used in vivo.

\section{METHODS}

\section{Tissue culture}

Inner ears. Swiss-Webster, CD-1, or C57BL/6 mouse embryos were harvested from euthanized, timedpregnant females at embryonic day (E) 12, 13, or 14. All methods were approved by the Oberlin College IACUC committee. The ears were dissected from all embryos in each litter (8-14 embryos) using fine forceps and were cleaned of as much of the surrounding mesenchyme and statoacoustic ganglion as possible. The approximate number of cells harvested from inner ears was 180,200 at E12; 216,900 at E13; and 344,500 at E14 (Table 1). Pooled inner ears from each litter were collected in serum-free culture medium [Dulbecco's Modified Eagle's Medium (DMEM)/F-12 supplemented with insulin, transferrin, selenious acid, and bovine serum albumin $\left(\right.$ ITS $^{+}$, Collaborative Research, Bedford, MA)]. The inner ears were rinsed three times in $\mathrm{Ca}^{2+} / \mathrm{Mg}^{2+}$-free phosphate buffered saline (PBS) then incubated in trypsin $\left(0.1 \%\right.$ in $\mathrm{Ca}^{2+} / \mathrm{Mg}^{2+}$-free PBS) for $10 \mathrm{~min}$ with DNase (80 units/mL; Sigma, Chemical Co., St. Louis, MO) added during the final $5 \mathrm{~min}$. The tissue was then rinsed in PBS and incubated in trypsin inhibitor $\left(0.1 \%\right.$ in $\mathrm{Ca}^{2+} / \mathrm{Mg}^{2+}$-free PBS) for $10 \mathrm{~min}$. Cells were then resuspended in the serum-free culture medium and triturated with a fire-polished pipette.

Cells were plated in the culture medium in 48-well, poly-D-lysine-coated dishes $\left(1 \mathrm{~cm}^{2}\right)$ and incubated at $37^{\circ} \mathrm{C}$ in a humid, $5 \% \mathrm{CO}_{2}$ incubator for 1-6 days. After 24 and $48 \mathrm{~h}$ in vitro, cellular aggregates were classified based on shape and cellular organization. Dissociated cells were initially plated at densities ranging from 300,000 to $1,000,000$ cells $/ \mathrm{cm}^{2}$. A value of 700,000 cells $/ \mathrm{cm}^{2}$ was found to be the optimal density for reaggregation, and all subsequent experiments were done at this density.

Limb bud cultures. To determine whether the aggregation patterns observed in inner ear cultures were common to all tissue types, E13 limb buds were used. Both fore- and hindlimbs were removed and prepared as above for dissociated inner ear cultures. Cells were plated at densities of 700,000 cells/well and processed for cytokeratin immunoreactivity (below) after $48 \mathrm{~h}$ in vitro.

\section{Immunocytochemistry}

Calretinin and parvalbumin 3. Cells were fixed in $10 \%$ neutral-buffered formalin for $10 \mathrm{~min}$. The cells were then treated with $0.3 \%$ hydrogen peroxide and blocked with $1 \%$ normal goat serum for $20 \mathrm{~min}$. Cells were incubated overnight in primary antibody [anticalretinn, $1 \mu \mathrm{g} / \mathrm{mL}$ (Chemicon, Temecula, CA) at $4^{\circ} \mathrm{C}$; anti pv3, 1:1,000 (Dr. Stefan Heller, Harvard MEEI) at room temperature]. Negative controls lacked primary antibody. The following day the cells were rinsed in PBS then incubated in secondary antibody [biotinylated goat antirabbit, 1:200 (Vector Labs, Burlingame, CA)] for $20 \mathrm{~min}$. The cells were rinsed and then incubated for $30 \mathrm{~min}$ with avidinbiotin horseradish peroxidase complex (ABC, Vector Labs). Sites of binding were visualized using diaminobenzidine-HCl (DAB, Sigma).

Myosin VIla. To further confirm that calretinin and pv3 immunopositive cells were hair cells, a third haircell-specific antibody was used. After $96 \mathrm{~h}$ in culture, cells were fixed in $4 \%$ paraformaldehyde for $1 \mathrm{~h}$. Cells were then incubated with $0.1 \%$ saponin and rinsed 3 times in PBS $/ 0.1 \%$ Tween for $30 \mathrm{~min}$. The cell cul- 
TABLE 1

Influence of embryonic age on aggregate formation and hair cell identification ${ }^{\text {a }}$

\begin{tabular}{|c|c|c|c|}
\hline & E12 & E13 & E14 \\
\hline Mean number of cells/inner ear & $180,203( \pm 14,172)$ & $216,859( \pm 26,504)$ & $344,494( \pm 21,780)$ \\
\hline \multicolumn{4}{|l|}{ Time of aggregate formation at density of: } \\
\hline 300,000 cells $/ \mathrm{cm}^{2}$ & None & None & ND \\
\hline 500,000 cells $/ \mathrm{cm}^{2}$ & None & $48 \mathrm{~h}$ & ND \\
\hline $700,000 \mathrm{cells} / \mathrm{cm}^{2}$ & None & $24 \mathrm{~h}$ & $24 \mathrm{~h}$ \\
\hline $1,000,000 \mathrm{cells} / \mathrm{cm}^{2}$ & None & $24 \mathrm{~h}$ & $24 \mathrm{~h}$ \\
\hline Time of prominent $\mathrm{HC}$ marker immunoreactivity & ND & 5 days in vitro & $3-4$ days in vitro \\
\hline
\end{tabular}

${ }^{a}$ The mean number of cells per inner ear $( \pm$ SE) was determined for each embryonic age by calculating the total number of cells per assay and dividing by the number of inner ears harvested. Aggregate formation was monitored for each embryonic age, and the time at which aggregate formation was first noted is listed for each density. The time at which hair cell immunoreactivity first became prominent is listed for each stage, indicating the time course for hair cell maturation in vitro. $\mathrm{ND}=$ not determined.

tures were then treated with $0.3 \%$ hydrogen peroxide and preblocked with $10 \%$ normal goat serum and $0.03 \%$ saponin. Cell cultures were incubated with antimyosin VIIa antibody $(10 \mu \mathrm{g} / \mathrm{mL}$; Dr. Tama Hasson, UCSD) at $4^{\circ} \mathrm{C}$ overnight. Negative controls lacked primary antibody. Cultures were rinsed with PBS/0.1\% Tween and incubated with secondary antibody [biotinylated goat antirabbit, 1:200 (Vector Labs) ] overnight at $4^{\circ} \mathrm{C}$. The next day they were incubated for $30 \mathrm{~min}$ in $\mathrm{ABC}$, rinsed, and developed using DAB (Sigma).

Cytokeratin. A monoclonal anticytokeratin antibody was used to detect nonsensory cells. The cultures were fixed for $10 \mathrm{~min}$ in $10 \%$ neutral-buffered formalin, rinsed in PBS, then treated with $0.3 \%$ hydrogen peroxide and blocked with $1 \%$ normal goat serum for $20 \mathrm{~min}$. Cultures were then incubated in anticytokeratin antibody (1:200, Sigma) for $2 \mathrm{~h}$ at room temperature. Cultures were rinsed in PBS, incubated in either biotinylated secondary antibody (1:200, Vector Labs), followed by treatment with ABC (Vector Labs) and visualized using DAB or a Texas Red-conjugated secondary antibody (Vector Labs) which was visualized by fluorescent optics. Double labeling for hair cells and supporting cells was conducted by incubating the cells in primary antibodies as described above, then treating them with fluorescent secondary antibodies. Calretinin was detected with a fluorescein-conjugated secondary antibody (1:400) and cytokeratin with the Texas Red-conjugated secondary antibody $(1: 200)$.

BrdU labeling of proliferating cells. To determine whether cells continued to proliferate in vitro, 5-bromo-2'-deoxyuridine (BrdU; $3 \mu \mathrm{g} / \mathrm{mL}$, Sigma) was added to the cultures at the time of plating, 2 days after plating, or 3 days after plating. BrdU was left in the cultures for $24 \mathrm{~h}$ and then removed, and cells were fixed in $10 \%$ neutral-buffered formalin for 20 min. Cultures were processed for BrdU immunocy- tochemistry following the methods of Warchol and Corwin (1996). Following fixation, cells were rinsed in PBS and treated with $90 \%$ methanol $/ 0.3 \%$ hydrogen peroxide for $30 \mathrm{~min}$ then $2 \mathrm{~N} \mathrm{HCl}$ for $30 \mathrm{~min}$. Cells were rinsed in PBS one time, then preblocked in $0.5 \%$ BSA and $2 \%$ normal goat serum for $30 \mathrm{~min}$. Cells were incubated in anti-BrdU antibody (1:50, BD Biosciences, San Jose, CA) in PBS with $1 \%$ triton and $2 \%$ normal goat serum overnight at $4^{\circ} \mathrm{C}$. The following day the cells were rinsed in PBS three times over $15 \mathrm{~min}$, then incubated in a biotinylated antimouse secondary antibody (1:200) for $2 \mathrm{~h}$ at room temperature. Cells were then rinsed three times in PBS for 15 min and incubated in the ABC complex, followed by development with DAB. The number of BrdUpositive cells in the confluent layer was counted in $2500 \mu \mathrm{m}$ fields selected from across each culture well. The number of fields counted depended on the particular cell sheet, as some curled during processing, limiting the areas that could be counted accurately. The number of proliferating cells was also counted in individual dome and thick-walled aggregates.

\section{Scanning electron microscopy}

For scanning electron microscopy (SEM) cells were grown on round glass coverslips coated with poly-Dlysine (BD Biosciences, Bedford, MA). Coverslips were placed in 24 well plates and cells were grown as described above for 3-4 days. Cell sheets typically began to lift off of the glass coverslips after 4 days in vitro, and therefore SEM cultures were maintained for a maximum of 4 days. After 3-4 days, the cells were fixed in $1 \%$ gluteraldehyde for $10 \mathrm{~min}$. Cells were rinsed with PBS three times over $20 \mathrm{~min}$ and then treated with $1 \%$ osmium tetroxide for $30 \mathrm{~min}$. Cells were rinsed with PBS and then dehydrated through ethanol (10\%-100\%, 10 min each). Cells were then incubated in equal volumes of $100 \%$ ethanol and acetone for $10 \mathrm{~min}$ and then 

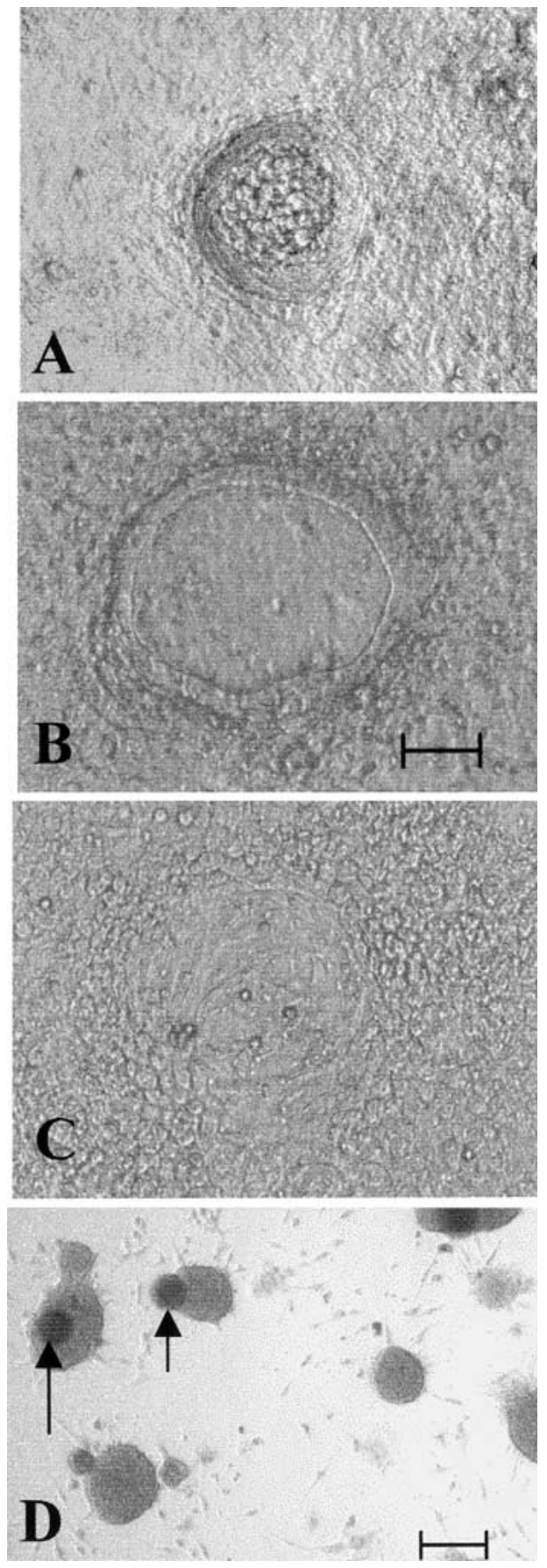

FIG. 1. Dissociated inner ear cells aggregated into various patterns. A-C. Examples of aggregate types that formed within $24-48 \mathrm{~h}$ in vitro. A. Dome aggregate. B. Thick walled aggregate. C. Flat aggregate. D. In contrast to inner ear cells, dissociated limb bud cells formed rounded masses surrounded by scattered cells which failed to form a confluent layer. Smaller clusters of cytokeratin-positive cells are located within some of the masses (arrows). Scale bars for A-C shown in $\mathbf{B}, 50 \mu \mathrm{m} ; \mathbf{D}, 50 \mu \mathrm{m}$.

$100 \%$ acetone for $10 \mathrm{~min}$. The cell sheets were then placed in a critical point dryer (LADD Research Industries, Williston, VT) and then sputter-coated with gold (LADD Research Industries). Cultures were viewed and photographed on a Jeol JSM5310 scanning electron microscope.

\section{Imaging}

All phase-contrast, bright-field, and fluorescent images were viewed on a Zeiss Axiovert S 100 microscope and were captured using a Zeiss Axiocam digital camera and associated Axiovision software.

\section{RESULTS}

\section{Aggregation in vitro}

The first goal of this study was to establish whether dissociated E12, E13, or E14 mouse inner ear cells aggregate on a two-dimensional substrate. Initially, E13 cells were plated at various densities to examine whether cell density influenced aggregation. E13 inner ear cells from a single litter were pooled and plated on poly-D-lysine-coated wells at densities of $300,000,500,000,700,000$, or 1,000,000 cells/well (1 $\mathrm{cm}^{2}$ ). Within $24 \mathrm{~h}$, cultures with 700,000 and $1 \times 10^{6}$ cells formed a confluent layer throughout the well with several aggregates of various shapes located within or on top of this confluent layer. Wells with a density of 500,000 cells did not show aggregation at 24 $\mathrm{h}$ but showed some aggregates by $48 \mathrm{~h}$. Cultures containing 300,000 cells did not demonstrate aggregation at 24 or $48 \mathrm{~h}$ (Table 1). Additionally, wells plated at densities below 700,000 cells were not confluent and displayed fewer aggregates than the higherdensity cultures. Because cultures with 700,000 cells/ $\mathrm{cm}^{2}$ were found to yield aggregates consistently within $24 \mathrm{~h}$ in vitro, this was chosen as the standard plating density for all subsequent experiments examining E13 and E14 inner ear cells. Aggregation was not observed in E12 inner ear cultures at any of the densities tested.

\section{Aggregate morphology}

Within the first 24 h, E13 and E14 inner ear cells began to form aggregates of distinct shapes. All cultures were examined immediately after plating to ensure that undissociated cell aggregates were not present. Any cultures with undissociated clumps were excluded from further analysis. Thus, all aggregation described is a result of reassociation of cells rather than failure of cells to be disrupted initially.

Examination of cultures by phase-contrast microscopy indicated that three primary aggregate shapes were consistently observed in cultures of E13 and E14 inner ear cells. All wells contained combinations of all three aggregates distributed throughout the wells. The "dome" aggregate were 

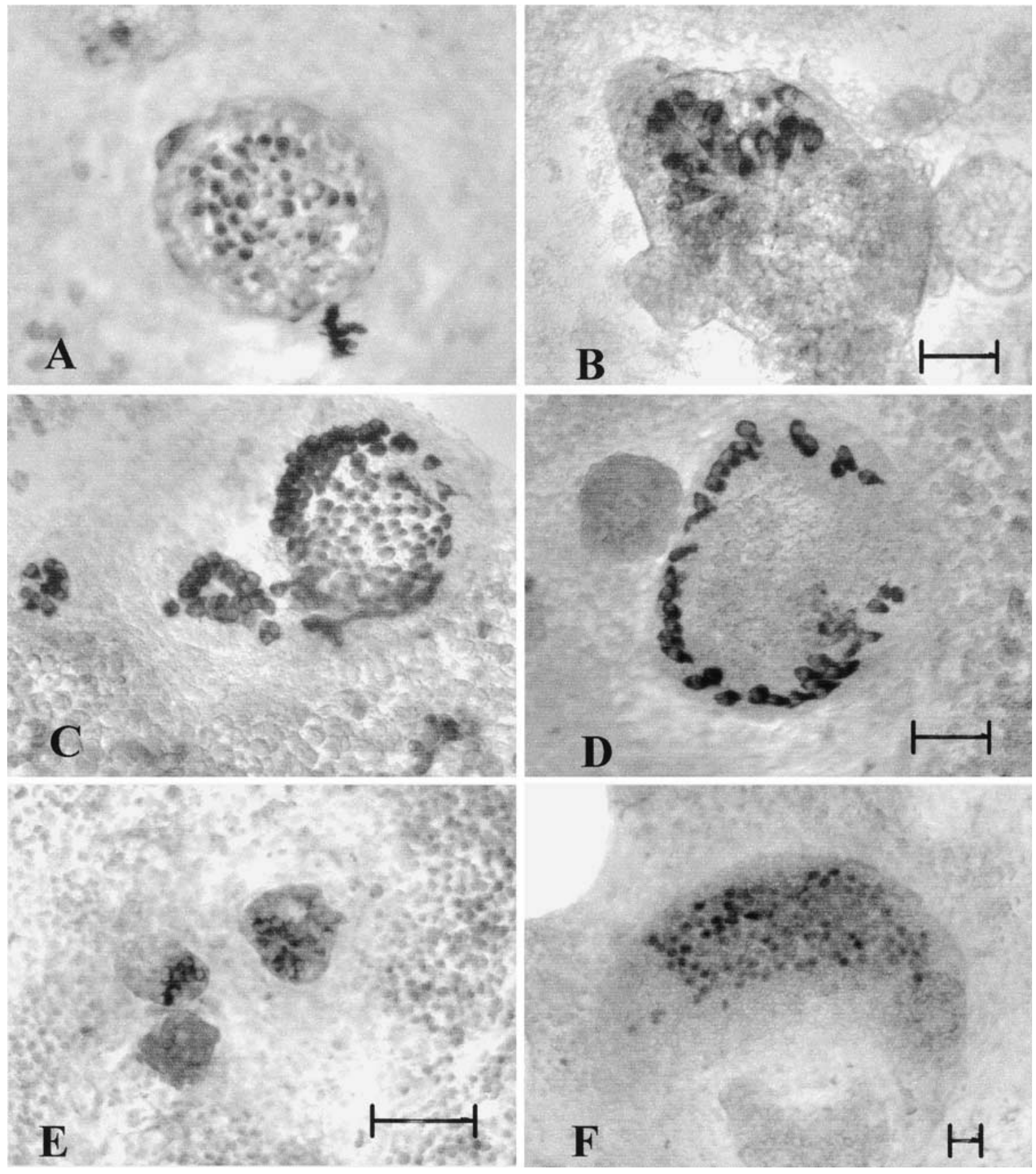

FIG. 2. Immunoreactivity for various hair cell markers (dark cells, A-F) was detected only in dome aggregates. A,B. Calretinin. C,D. Myosin VIla. E, F. Pv3. Hair cells were organized in various patterns including circular clusters $(\mathbf{A}, \mathbf{C}, \mathbf{F})$, rosettes $(\mathbf{B}, \mathbf{C}, \mathbf{E})$, or lines $(\mathbf{D})$. Scale bars $=50 \mu \mathrm{m}$.

characterized by an outer rim and apparent raised center (Fig. 1A). "Thick-walled" aggregate were characterized by a thick outer wall of epithelial-like cells and an inner layer of smooth cells (Fig. 1B). Both of these aggregate types were detected within 24-48 $\mathrm{h}$ in vitro. The number of each aggregate type varied within each well. Typically, the number of dome and thick-walled aggregates each ranged from 3 to $20 \mathrm{~cm}^{2}$. The third aggregate type, the "flat" aggregates, was characterized by a thin rim and central region of flattened cells organized in a different pattern than the surrounding confluent layer (Fig. 1C). These aggregates were typically observed after $48 \mathrm{~h}$ in vitro.

Most aggregates were circular or oval in appearance, though some thick-walled aggregates had more irregular shapes. The diameter of dome aggregates ranged between 100 and $300 \mu \mathrm{m}$ and the diameter of thick-walled aggregates ranged between 200 and 400 $\mu \mathrm{m}$ after $24 \mathrm{~h}$. The diameter of individual aggregates 
did not increase at later time points in vitro (up to 6 days in vitro).

\section{Limb bud cultures}

To further test whether the aggregation observed from embryonic inner ear cells represented a specific patterning mechanism or just a random association of cells, another tissue was processed using the same methods and plating density. Limb buds were chosen as they are easily accessible, contain a mixture of cell types, and are a self-organizing tissue (Tabin 1995). When E13 limb buds were cultured under these conditions, a remarkably different pattern of aggregation was observed (Fig. 1D). Unlike inner ear cells, limb bud cells did not form a confluent layer of cells across the substrate, even after $48 \mathrm{~h}$ in vitro. Limb bud cells formed large, round masses throughout the well surrounded by individual cells. Immunocytochemical staining with anticytokeratin antibodies revealed light immunoreactivity throughout the cell masses, with areas of more intense reactivity confined to clusters of cells within some of the masses (Fig. 1D). Thus, limb bud cells and inner ear cells behave very differently when cultured under the same conditions.

\section{Localization of hair cells in vitro}

The majority of cultured inner ear cells were unidentifiable solely on appearance using phase-contrast microscopy. Occasional, neuronlike cells were observed; however, these were rare as care was taken to remove as much ganglia as possible prior to dissociation. In order to identify the cell types associated with the aggregates, immunocytochemical studies were completed using anti-calretinin, anti-myosin VIIa, and anti-parvalbumin 3 antibodies, all of which have been reported to be specific for hair cells (Dechesne et al. 1994; Hasson et al. 1997; Heller 2001).

Cells immunopositive for calretinin were located only in aggregates with dome morphology (Fig. 2A, B). Other aggregate types were not immunopositive for calretinin. No staining was observed in controls lacking primary antibody. Immunopositive cells were often seen adjacent to regions of unreactive cells. Approximately $70 \%$ of dome aggregates contained cells immunopositive for calretinin ( $n=8$ wells). Within dome aggregates, hair cells were observed in diverse patterns. Calretinin-positive cells were often arranged in circular groups, rosettes, lines, or stripes. Occasionally, single hair cells were located in patches of nonimmunoreactive cells. Similar patterns of immunoreactivity were detected with myosin VIIa (Fig. 2C,D) and pv3 (Fig. 2E,F), demonstrating that these markers detected hair cells in dissociated cell cultures
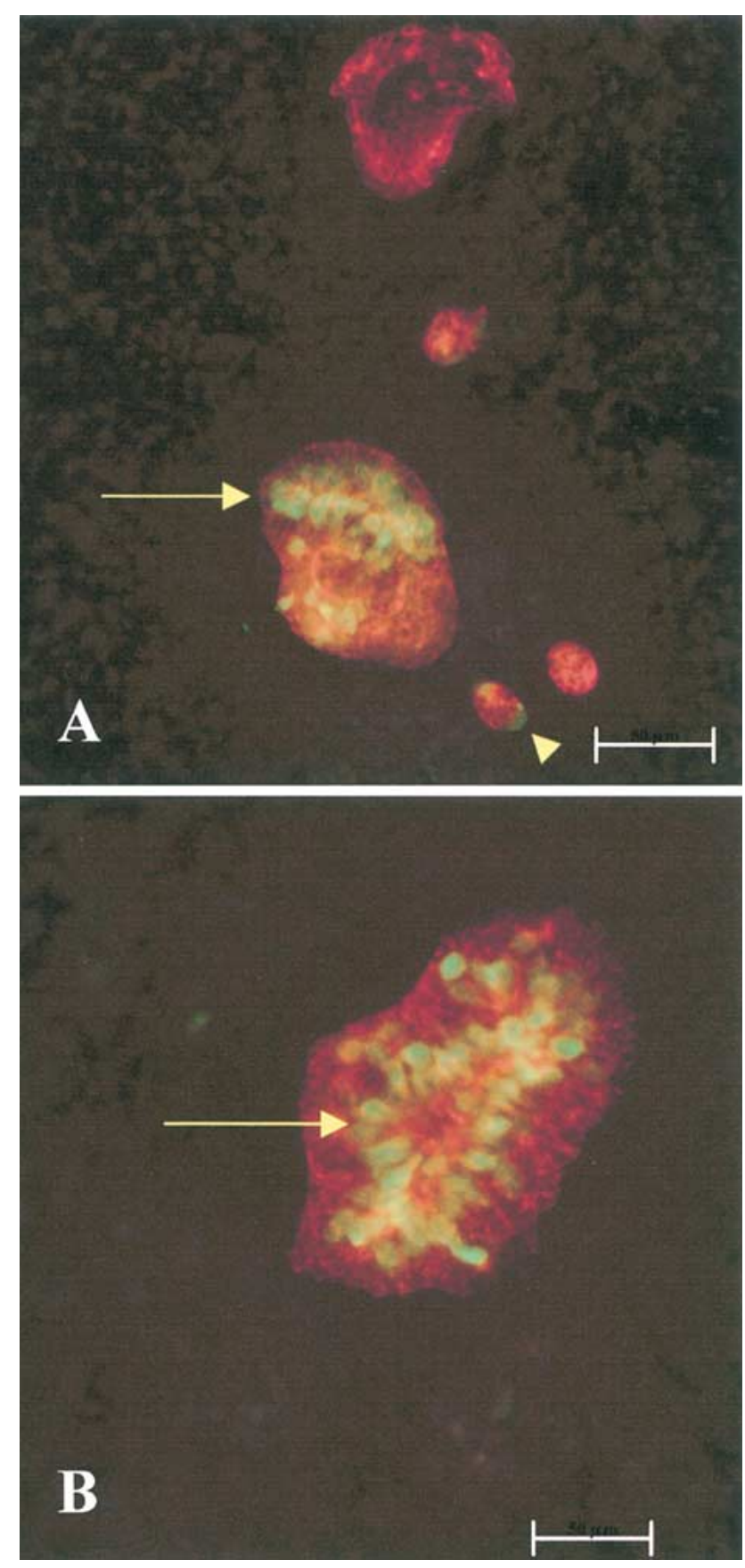

FIG. 3. Hair cells and supporting cells were colocalized in dome aggregates. A. Cytokeratin-positive nonsensory cells (red) were localized to both dome (arrow) and thick-walled aggregates (top image, red). Only dome aggregates contained calretinin-positive hair cells (green). Hair cells were surrounded by supporting cells in dome aggregates (arrow). B. Calretinin-positive hair cells (green) in the central region of a dome aggregate surrounded by cytokeratin-posi tive nonsensory cells. This aggregate is similar in appearance to the one shown in Figure 4A. Scale bars $=50 \mu \mathrm{m}$.

as well as in vivo. The similarity of patterning further indicated that hair cells reassociated into specific patterns. The number of hair cells per dome aggregate ranged from 8 to 193 (mean 53; $n=19$ domes), 

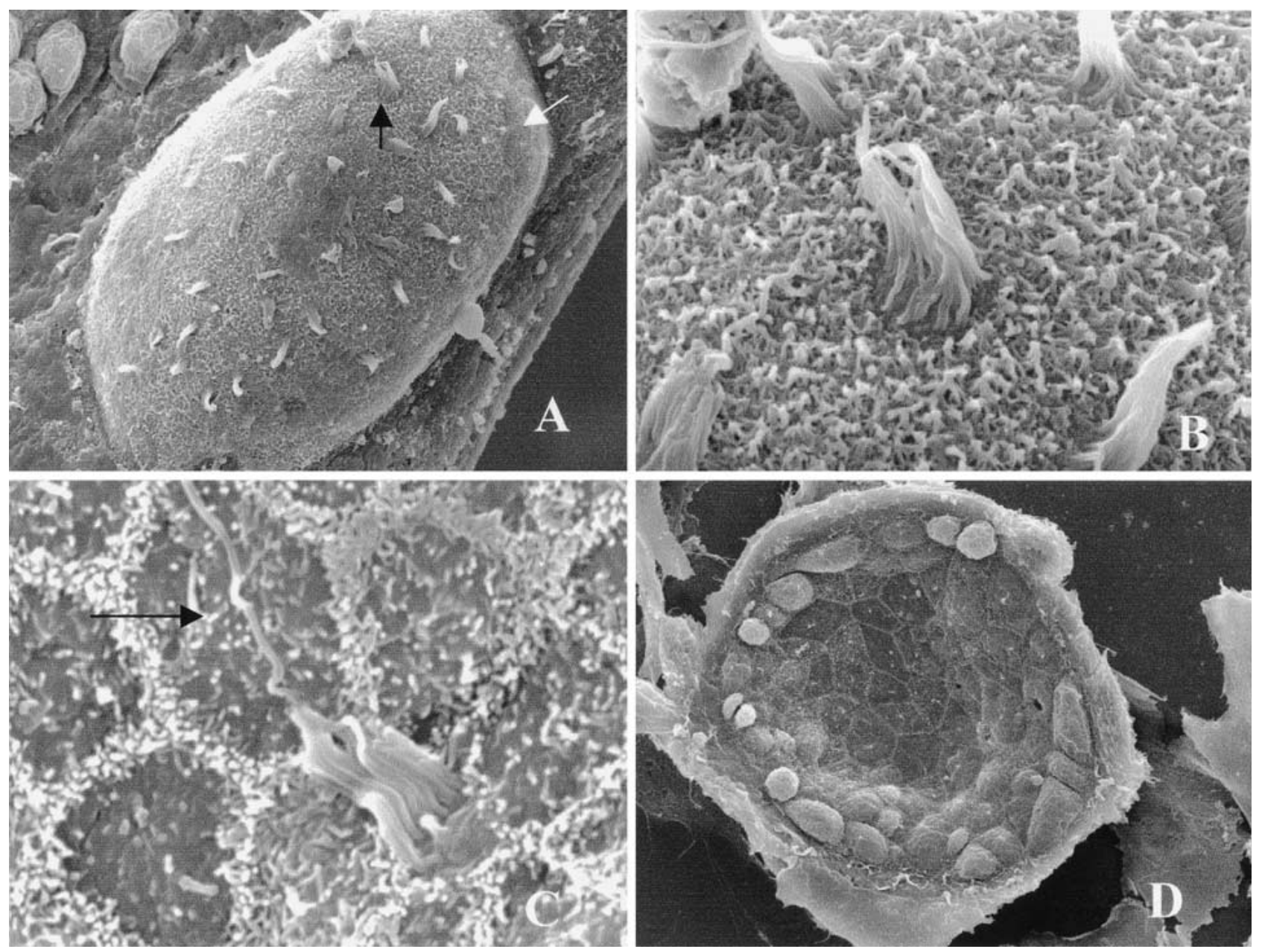

FIG. 4. Scanning electron micrographs of cell aggregates. A. Dome aggregate, similar to the one shown in Figure 3B. Stereocilia-bearing hair cells are located centrally on the top of the aggregate (black arrow), while supporting cells are clearly identifiable on the side of the aggregate (white arrow). B. Closeup of a stereocilia bundle

depending on the size of the aggregate and the pattern of hair cells within the aggregate.

\section{Localization of supporting cells in vitro}

In order to determine where nonsensory supporting cells were located in relation to hair cells, an anticytokeratin antibody was used to identify nonsensory cells. Cytokeratin-positive cells were located within both dome and thick-walled aggregates (Fig. 3A,B). None of the flat circle aggregates, identified by phase-contrast microscopy (Fig. 1C) were immunoreactive for cytokeratin or hair cells markers. Within the dome aggregates, cytokeratin-positive cells were located in regions complementary to cells positive for hair cell markers (Fig. 3A,B). The rim of dome aggregates was typically immunoreactive for cytokeratin, as were cells within the central region of the aggregate. Cell borders were often

shown in A (black arrow). C. Streocilia bundle with a long process reminiscent of a kinocilium (arrow). The bundle is surrounded by supporting cells. D. Thick-walled aggregate composed of a central concave region of support-like cells and a raised rim of additional nonsensory cells.

distinctly outlined by the cytokeratin marker within the aggregates.

\section{Colocalization of hair cells and supporting cells in dome aggregates}

Double labeling for supporting cells and hair cells indicated that cytokeratin-positive cells were typically adjacent to or below hair cells (Fig. 3). No cells were double labeled for both hair cell and nonsensory cell markers. By focusing through the double-labeled aggregates, it appeared that hair cells were arranged on top of the central region of cytokeratin-positive cells.

SEM analysis confirmed that hair cells were located on top of the domes and were surrounded by supporting cells. Hair cells were identified by the presence of stereocilia. The majority of domes contained only scattered stereocilia-bearing cells, whereas a few were covered with stereocilia. In domes covered with 


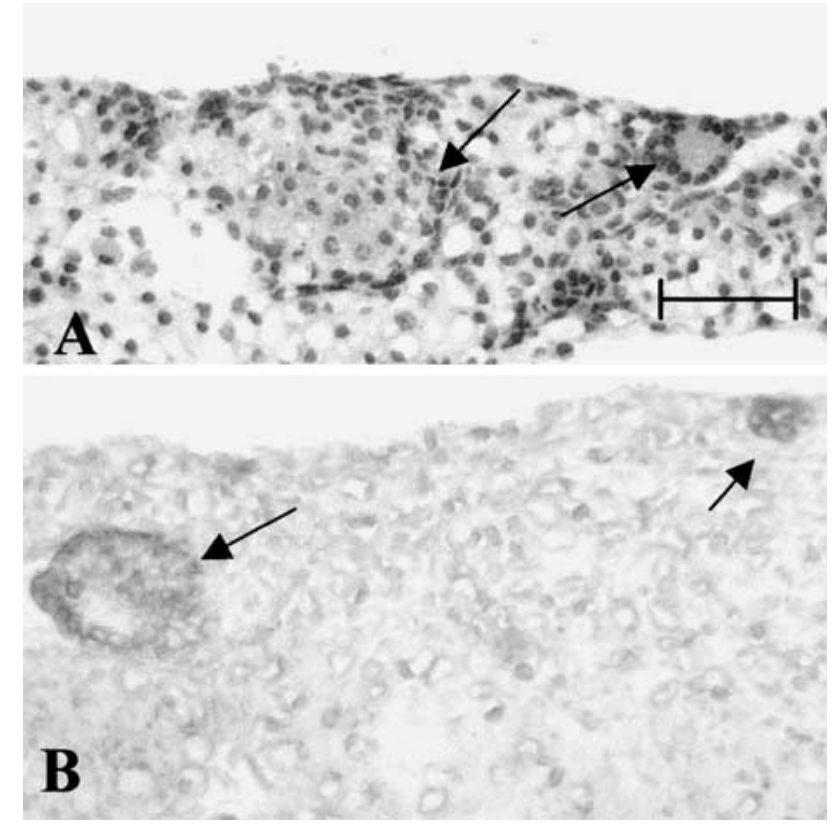

FIG. 5. A cross section through the upper portion of a cell sheet stained with hematoxylin and eosin revealed that cellular aggregates were solid masses rather than hollow spheres (arrows). B. Cytokeratin-positive cells are observed within some of the cell aggregates (arrows). Scale bar $=50 \mu \mathrm{m}$.

stereocilia, the stereocilia-bearing cells were located on the top of the aggregate (Fig. 4A) and each cell was surrounded by short microvilli (Fig. 4A,B). The spacing between stereocilia-bearing cells suggested that other cells, such as supporting cells, surrounded the hair cells. In addition, cells characteristic of supporting cells, as previously observed in intact organ of Corti, were located along the sides of the dome (Fig. $4 \mathrm{~A})$. In other aggregates expressing fewer stereociliabearing cells, single hair cells were surrounded by several presumptive supporting cells (Fig. 4C). Thus, SEM revealed hair cells and supporting cells coexisting in the dome aggregates.

Most of the stereocilia on the aggregates were bundles without any clear orientation, kinocilium, or height gradient. Therefore, they may represent immature cochlear or vestibular stereocilia. However, all of the stereocilia observed in the present study were similar in appearance to those shown in Figure 4B. No stereocilia bundles were organized in a shape typical of cochlear inner or outer hair cells. Occasional bundles possessed a projection that was longer than the surrounding stereocilia, projections that were reminiscent of kinocilia (Fig. 4C).

Thick-walled aggregates were also detected by SEM (Fig. 4D). Consistent with immunocytochemical studies, these aggregates did not contain hair cells. The concave central region consisted of numerous cells whose appearance was typical of supporting cells previously observed by SEM in whole-mount preparations.
To determine whether the aggregates represented hollow balls or solid masses of cells, cell sheets were removed from the wells, embedded in paraffin, and sectioned at $4 \mu \mathrm{m}$. The sections revealed that the cell sheets contained solid masses of cells. Many of the cells were organized in distinct clusters (Fig. 5A). Because the hair cell markers used in the present study did not work in paraffin-embedded tissues, it was not possible to determine the location of hair cells in these cell sheets. The anticytokeratin antibody labeled circular aggregates in the cell sheets indicating areas of nonsensory cells (Fig. 5B). Whether these aggregates correspond to the dome or thick-walled aggregates observed by phase-contrast and scanning electron microscopy could not be determined.

\section{Maturation of hair cells in vitro}

In order to investigate whether hair cells continued to mature in inner ear cultures, the time course of calretinin immunoreactivity was examined. In E13 cultures, immunoreactivity was absent after 2 days in vitro. Prominent immunoreactivity was not detected until 5 days in vitro (Table 1). Immunoreactivity of E14 inner ear cultures showed weak immunoreactivity after 2 days in vitro, but prominent immunostaining was detected by 3-4 days in vitro (Table 1 ). Immunoreactivity continued to be detected until 6 days in vitro, the latest time point examined. Assuming that hair cell maturation continues in vitro, the observed increased immunoreactivity would correspond to approximately E18-20, a period at which hair cells express calretinin immunoreactivity in vivo.

Because pv3 detects hair cells very early in chick and may therefore be able to detect hair cells earlier than the other hair cell markers, immunoreactivity of E14 mouse inner ear cells was tested at 1, 3, and 5 days in vitro. No immunoreactivity was noted after 1 day in vitro, but immunoreactivity was clearly detected by 3 days in vitro, similar to that observed with calretinin (Table 1). Thus, E14 inner ear cells appear to begin to express hair-cell-specific proteins after approximately 3 days in culture.

Further evidence that hair cells mature in vitro came from examination of cultures by SEM. It was noted that few stereocilia-bearing cells were present after 3 days in culture, but several were detected by 4 days in culture, the latest time point examined by SEM. Together, these observations suggest that both hair cell proteins and stereocilia mature in vitro.

\section{Proliferation of cells in vitro}

In order to determine whether cells continued to proliferate in vitro, BrdU was added to cultures of E14 
TABLE 2

Mean number of BrdU-positive cells in cultures ${ }^{\mathrm{a}}$

\begin{tabular}{lccc}
\hline & \multicolumn{3}{c}{ BrdU-positive cells } \\
\cline { 2 - 4 } Region of proliferating cells & day $0-1$ & day 2-3 & day 3-4 \\
\hline Fields of the confluent layer & $598( \pm 15)$ & $382( \pm 24)$ & $240( \pm 32)$ \\
Dome aggregates & $24( \pm 7)$ & $46( \pm 10)$ & $28( \pm 5)$ \\
Thick-walled aggregates & $20( \pm 2)$ & $37( \pm 4)$ & $27( \pm 6)$ \\
\hline
\end{tabular}

${ }^{\mathrm{a}} \mathrm{BrdU}$ was added for a $24 \mathrm{~h}$ period beginning at the time of plating (day 0), 2 days after plating, or 3 days after plating. The mean number of BrdU-positive cells ( \pm standard error) in the confluent layer is based on the number of proliferating cells in a $2500 \mu \mathrm{m}$ area (5-10 fields per culture period). The mean number of proliferating cells ( \pm standard error) was also counted in individual dome and thick-walled aggregates (3-6 aggregates per culture period).

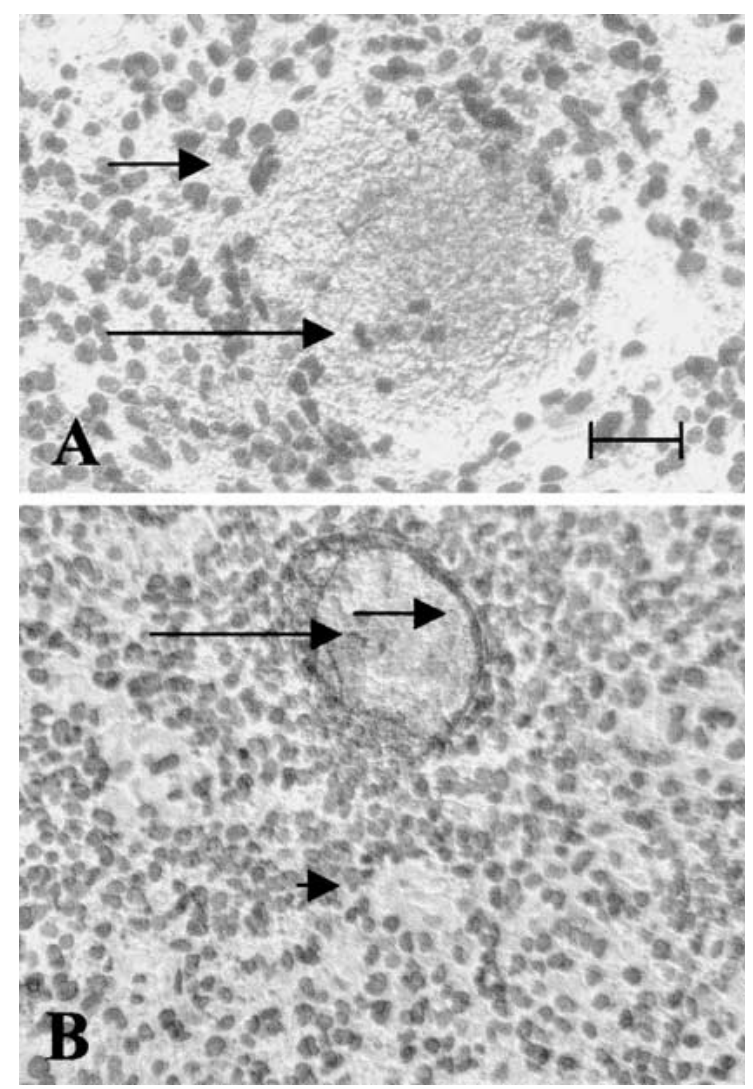

FIG. 6. BrdU-positive cells were located primarily in the unidentified cells of the confluent layer (A, B, dark cells). Occasional BrdUpositive cells were found in the central portion of dome aggregates (A, large arrow) or thick-walled aggregates (B, large arrow), but most of the proliferating cells associated with these aggregates were localized to the outer rims of these aggregates (A, B, small arrows). Proliferating cells were rarely seen within the central region of flat aggregates (arrowhead). Scale bar $=50 \mu \mathrm{m}$.

inner ear cells at the time of plating, $48 \mathrm{~h}$ after plating, or $72 \mathrm{~h}$ after plating. The BrdU was left in for $24 \mathrm{~h}$, then cells were processed for BrdU immunocytochemistry. The proliferating cells were located primarily throughout the confluent layer of cells (Table 2), cells that were not immunoreactive for hair cell or nonsensory cell markers. Thus, the identity of these cells is unknown. The number of proliferating cells in the confluent layer decreased over time in culture. BrdU-positive cells were also seen in thickwalled and dome aggregates, but these represented less than $10 \%$ of the total number of proliferating cells (Table 2). In dome aggregates, the BrdU-positive cells were mainly located around the rim of the dome, an area that contains nonsensory supporting cells (Fig. 6A). Thick-walled aggregates had labeled cells around the rim as well as in the central region (Fig. 6B). The flat-circle aggregates showed few or no BrdU-positive cells in the central region of cells, but proliferating cells were seen all along the outer edge of these aggregates (Fig. 6B). Double labeling with calretinin and BrdU did not reveal cells that were positive for both markers (not shown).

\section{DISCUSSION}

\section{Aggregation of inner ear cells}

The embryonic inner ear gives rise to a diverse and complex set of specialized cells during development. The precise arrangement of these cells is critical for correct functioning of the cochlear and vestibular organs. A potentially useful model with which to study the roles of molecular cues in vitro utilizes the natural ability of cell types to reaggregate following dissociation. Reaggregation of cells has been studied in a number of cell types using a variety of culture methods, such as rotating cultures, pellet cultures, and monolayer cultures. The ability of specific cell types to reaggregate has been demonstrated in many tissues, including the chick inner ear (Orr 1968). Although these early studies demonstrated that dissociated $\mathrm{E} 4$ chick otocyst cells reaggregated into specific cell groups, including epithelial-like structures, the identity of the cell types could not be determined due to the lack of available markers at that time.

In the present study, mammalian inner ear cells were placed on a two-dimensional surface to see if precise patterning could still take place outside of the normal three-dimensional environment. Inner ear cells from E12-14 mouse inner ears were dissociated, placing all cell types of the developing vestibular 
andcochlear regions in a random arrangement. The entire ear was used to see if the cells from different regions and tissue types would be able to sort out into particular cell groupings. The cells were plated onto poly-D-lysine-coated dishes and allowed to settle. The cells formed distinct aggregates within the first $24 \mathrm{~h}$ in vitro. Monitoring of cells at various time points from 2 to $24 \mathrm{~h}$ in vitro indicated that cells were fully dissociated initially and the observed cell patterns were not a result of undissociated clumps of tissue.

\section{Hair cell patterning}

Three distinct aggregate forms were recognized in each culture preparation. Significantly, only the dome aggregates contained hair cells as demonstrated by immunocytochemical studies using three different hair-cell-specific antibodies (anti-calretinin, anti-pv3, and anti-myosin VIIa antibodies) and SEM analysis. These results indicated that mammalian hair cells organize into specific patterns in vitro.

The patterns of hair cell organization within the aggregates varied somewhat within each aggregate, yet all three markers picked up these same patterns, indicating that the markers detected hair cells in vitro as well as in vivo. Hair cells were typically organized as circles, stripes, lines, or rosettes surrounded by regions of nonsensory cells. The significance of the different hair cell patterns is not yet known but may reflect differences in the organization of cochlear and vestibular epithelia. However, because the available hair cell markers are unable to differentiate cochlear from vestibular hair cells, the present study could not address whether particular patterns are associated with different sensory regions of the inner ear.

\section{Organization of supporting cells and hair cells}

In dome aggregates, cytokeratin-positive cells typically appeared adjacent to hair cells, suggesting that hair cells and supporting cells colocalize in one aggregate subtype. The pan-cytokeratin antibody used in the present study had previously been used to identify nonsensory cells in the inner ear (Stone et al. 1996). Although not intended to be a specific marker of a given supporting cell type, the cytokeratins label supporting cells of the sensory epithelia beginning in embryonic development as well as cells of the stria vascularis (Kuijpers et al. 1991, 1992). Cytokeratins are found only in epithelial cells and not mesenchymal cells. Cytokeratin-immunopositive cells were often localized in the outer rim of the dome aggregate as well as below the layer of hair cells. The labeling of nonsensory cells in close association with hair cells suggests that the cells are not likely to be from the stria vascularis, though this possibility cannot be ruled out based on the current data. Analysis by SEM further revealed cells characteristic of supporting cells located between stereocilia bundles and around the rim of the dome aggregates. Thus, in vitro, hair cells and supporting cells organize into distinct patterns, similar to the patterns found in vivo. No cells appeared to be double labeled with hair cell and supporting cell markers. Although no hair cells were noted to be in contact with one another or clustered in areas without supporting cells, it is possible that some hair cells were in direct contact with one another. Such direct contact has been noted in developing avian inner ears and the inner ears of mice lacking jag2, a member of the notch signaling family (Goodyear and Richardson 1997; Lanford et al. 1999). However, in the present study, any such direct contacts would be rare, as the majority of hair cells were surrounding by supporting cells as observed by immunocytochemistry and SEM.

Thick-walled aggregates were also immunoreactive for cytokeratin, but these were not positive for hair cell markers. Thus, supporting cells appear to aggregate into various forms, whereas hair cells associate only with the dome aggregate. Due to the lack of specific supporting cell markers, it is currently not possible to identify the types of supporting cells localized to the various aggregate types.

\section{Limb bud cells have different aggregation patterns}

The aggregate forms observed from cultures of embryonic inner ear cells were specific to ear tissue. When limb bud tissue was cultured under the same conditions and at the same density, the cells behaved very differently. Rather than forming a confluent layer with distinct aggregate types located on top, limb bud cells failed to form a confluent layer. Large rounded masses were found throughout the well with smaller clusters of cytokeratin-positive cells located within the masses. The lack of similarity between the behaviors of these two tissues in vitro further indicates that inner ear cells are using specific mechanisms to regulate the patterning that occurs in vitro.

\section{Hair cell maturation in vitro}

All three of the hair cell markers used in the present study label hair cells soon after differentiation. Cultures of E13 and E14 inner ear cells began to express hair cell markers after 3-4 days in vitro. Studies examining the time course of calretinin and pv3 expression in vitro revealed that the time of expression of these hair cell markers was similar to the in vitro condition, suggesting that hair cells continue to mature in vitro. In the present study, hair cells from E13 mice did not express calretinin after 2 days in vitro. 
Prominent expression was observed by 5 days in vitro. In E14 inner ear cell cultures, calretinin and pv3 immunoreactivity was detected approximately 1-2 days earlier. Thus, the time course of expression in vitro would correspond to days E18-20 in vitro and is similar to that noted in vivo (Dechesne et al. 1994).

Stereocilia normally form between E13.5 and 15 in vivo (Lim and Anniko 1985). SEM revealed that stereocilia number increased between 3 and 4 days in vitro, further suggesting that hair cells mature in vitro. Previous reports of organ cultures of the entire otocyst found that the entire cristae was able to mature in vitro (Anniko et al. 1979). In that study, the maturation of hair cells and stereocilia tended to lag from one half day to one day behind that observed in vivo. Alternatively, in the present study, immature stereocilia damaged during the dissociation process may have recovered by this time. Overall, the number of domes covered with stereocilia were few. This may reflect differences in the timing of stereocilia maturation or recovery in the various domes. Because the cells formed sheets of confluent cells that began to lift off the substrate after 5 days in vitro, SEM could not be performed on cultures incubated beyond 4 days. Therefore, it was not possible to determine whether additional stereocilia form after 5 days in vitro.

\section{Proliferation of cells in vitro}

In the present study, cell proliferation continued from the time of plating through 4 days in vitro, the latest time point examined. Proliferation was primarily in the unidentified cells forming the confluent layer. These cells were not immunoreactive for hair cell or supporting cell markers. The number of proliferating cells per area of confluent cells evaluated decreased over the culture period. In contrast to the extensive proliferation in the confluent layer of cells, few BrdUpositive cells were located in dome, thick-walled, or flat aggregates. In dome or thick-walled aggregates, the number of BrdU-positive cells made up less than $10 \%$ of the total number of proliferating cells in the cultures. In dome aggregates, BrdU-positive cells were located mainly around the outer rim, in the area of nonsensory supporting cells. Thick-walled aggregates contained BrdU-positive cells along the outer rim as well as in the central region. The number of positive cells was influenced in part by the size of the aggregates, making it difficult to conclude whether cell proliferation changed over the course of the culture period. Although a slight increase in proliferation was observed after 2-3 days in culture in both of these aggregate types, this is most likely a reflection of variation in aggregate size rather than proliferation rates. A previous study comparing calretinin immunoreactivity and BrdU labeling indicated that calreti- nin is detected only in postmitotic cells and not in mitotic progenitors (Zheng and Gao 1997). These results are in agreement with the present study in which we were unable to identify any cells that were labeled with both calretinin and BrdU. Because the various aggregate types did not increase in size during the culture period, the proliferating cells do not appear to migrate into the aggregates over time.

The ability of inner ear cells to form distinct shapes and the observation that only one aggregate type contained hair cells suggested that some sort of cellcell recognition or fate-determining mechanism must be taking place. Further, the observation that E12 cells did not reaggregate suggests that these mechanisms are developmentally regulated. In other systems it has been proposed that reaggregates are formed by differential adhesion (causing the most adhesive cells to recognize each other) or specific adhesion (cell-cell recognition of specific adhesive molecules on the surface of cells; reviewed by Laylor and Willbold 1993). A general adhesive mechanism causes the most adhesive cells to establish contacts first and, therefore, would be centrally located with the less adhesive cells arranged around the perimeter (reviewed by Laylor and Willbold 1993). The observation that hair cells are found in specific patterns (i.e., rosettes, lines, stripes, single cells) throughout the aggregates suggests that a specific adhesion mechanism is involved.

Several membrane-bound molecules are present in the developing inner ear, including neural cell adhesion molecules (NCAM; Whitlon et al. 1999), cadherins (Whitlon et al. 1999; Petit 2001), integrins (Littlewood-Evans and Muller 2000), members of the Eph family (Bianchi and Gale 1998; Bianchi and Liu 1999), and members of the Notch/Delta family (Lanford et al. 1999; Shailam et al. 2000; Eddision et al. 2000). Whether any of these molecules are associated with cell recognition, fate determination, or aggregation in vitro remains to be determined. It is also unclear whether inner ear cells maintain their fate following dissociation or whether they develop as hair cells and supporting cells once they aggregate. By E14, the cells in the more basal turns of the cochlea are likely committed to their fate, whereas cells in the middle and upper turns are not yet committed to a particular cell fate (reviewed by Kelley and Bianchi 2001). Thus, in the current assay, there is likely a mix of cells that are committed to hair cell or supporting cell fate, as well as precursors that are not yet committed. Therefore, the aggregates may represent clusters of hair cells and supporting cells that use cell surface molecules to recognize one another and bind together into a particular pattern. Conversely, the cells may represent uncommitted cells that bind together then use cell surface molecules to determine cell fate. For example, members of the 
notch signaling pathway are important for establishing the normal patterning of hair cells and supporting cells in cochlear and vestibular epithelium (Lanford et al. 1999; Shailam et al. 2000; reviewed by Kelley and Bianchi 2000). Whether this family is important for the patterning observed following the aggregation that takes place in vitro is unknown. Future studies using this established cell culture system will be able to address such questions.

In summary, this study reveals that embryonic inner ear cells reassociate into a specific pattern when dissociated and placed in culture. The cells are able to sort out into these patterns despite the fact that they originate from such a complex mixture of cell types. The aggregation patterns are specific to inner ear cells and reflect patterns similar to those observed in vivo. This study not only demonstrates intrinsic cell patterning of inner ear cells, it also demonstrates a valuable assay for future studies to address the mechanisms underlying inner ear cell patterning.

\section{ACKNOWLEDGMENTS}

This study was supported by grants from the National Science Foundation (NSF) (IBN 9904566) and the Deafness Research Foundation (L.M.B.). The authors wish to thank Dr. S. Heller (MEEI) for the pv3 antibody, Dr. T. Hasson (UCSD/Yale) for the myosin VIIa antibody, and Dr. M. Kelley for his many helpful discussions on this manuscript.

\section{REFERENCES}

Anniko M. Nordemar H, Van De Water TR. Embryogenesis of the inner ear I: Development and differentiation of the mammalian crista ampullaris in vivo and in vitro. Arch. Otorhinolaryngol. 224:285-299, 1979.

BiANCHI LM, GALE NW. Distribution of Eph-related molecules in the developing and mature cochlea. Hear. Res. 117:161-172, 1998.

BiAnCHI LM, Liu H. Comparison of ephrin-A ligand and EphA receptor distribution in the developing inner ear. Anat. Rec. 254:127-134, 1999.

Dechesne CJ, Rabejac D, Desmadryl G. Development of calretinin immunoreactivity in the mouse inner ear. J. Comp. Neurol. 346:517-529, 1994.

EDdison M, Le Roux I, Lewis J. Notch signaling in the development of the inner ear: Lessons from Drosophila. Proc. Nat. Acad. Sci. USA 97:11692-11699, 2000.

Flock A, Bretscher A, Weber K. Immunocytochemical localization of several cytoskeletal proteins in inner ear sensory and supporting cells. Hear. Res. 6:75-89, 1982.

GoOdYEAR R, Richardson G. Pattern formation in the basilar papilla: Evidence for cell rearrangement. J. Neurosci. 17:62896301, 1997.
Hasson T, Gillespie PG, Garcia Ja, MacDonald RB, Zhao Y, Yee AG, MoOseker MS, Corey DP. Unconventional myosins in innerear sensory epithelia. J. Cell. Biol. 137:1287-1307, 1997.

Heller S. Parvalbumin 3 is a major mobile $\mathrm{Ca}^{2+}$ buffer of bullfrog inner ear cells. Abstr. Assoc. Res. Otolarygol. 24:42, 2001.

Kasper M, Stosiek P, VArga A, Karsten U. Immunohistochemical demonstration of the co-expression of vimetin and cytokeratin $(s)$ in the guinea pig cochlea. Arch. Otorhinolaryngol. 244:66-68, 1987.

Kelley MW, BiANChi LM. Development and neural innervation of the organ of Corti. In: Willet J, (ed) Handbook of Auditory Research. Boca Raton, CRC Press, pp. 137-156, 2001.

Kujpers W, Peters TA, Tonnaer EL, Ramaekers FC. Expression of cytokeratin polypeptides during development of the rat inner ear. Histocytochemistry 96:511-521, 1991.

Kujjpers W, Tonnaer ElGM, Peters TA, Ramaekers FCS. Developmentally-regulated coexpression of vimentin and cytokeratins in the rat inner ear. Hear. Res. 62:1-10, 1992.

Lanford P, Yu L, Jiang R, Lindsell C, Weinmaster G, Gridley T, KelLey M. Notch signaling pathway mediates hair cell development in mammalian cochlea. Nat. Genet. 21:289-292, 1999.

Lawlor P, Marcotti W, Rivolta MN, Kros CJ, Holley MC. Differentiation of mammalian vestibular hair cells from conditionally immortal, postnatal supporting cells. J. Neurosci. 19:9445-9458, 1999.

LAYlor PG, Willbold E. Histogenesis of avian retina in reaggregation culture: From dissociated cells to laminar neuronal networks. Int. Rev. Cytol. 146:1-46, 1993.

Lim DJ, Anniko M. Developmental morphology of the mouse inner ear. A scanning electron microscopic observation. Acta Otolaryngol. Suppl. 422:1-69, 1985.

LitTlewood-Evans A, Muller U. Stereocilia defects in the sensory hair cells of the inner ear in mice deficient in integrin alpha8betal. Nat. Genet. 24:424-428, 2000.

ORR MF. Histogenesis of sensory epithelium in reaggregates of dissociated embryonic chick otocysts. Dev. Biol. 17:30-45, 1968.

Petit C. Usher Syndrome: From genetics to pathogenesis. Annu. Rev. Genomics Hum. Genet. 2:271-297, 2001.

Self T, Mahony M, Fleming J, Walsh J, Brown SD, Steel KP. Shaker-1 mutations reveal roles for Myosin VIIa in both development and function of cochlear hair cells. Development 125:557-566, 1998.

Shailam R, Lanford PJ, Dolinsky CM, Norton CR, Gridley T, KELLEY M. Expression of proneural and neurogenic genes in the embryonic mammalian vestibular system. J. Neurocytol. 28:809819, 1999.

Stone JS, Leaño SG, Baker LP, Rubel EW. Hair cell differentiation in chick cochlear epithelium after aminoglycoside toxicity: In vivo and in vitro observations. J. Neurosci. 16:6157-6174, 1996.

TABIN C. The initiation of the limb bud: Growth factors, Hox genes and retinoids. Cell 80:671-674, 1995.

WARCHOL ME, CORWIN JT. Regenerative proliferation in organ cultures of the avian cochlea: Identification of the initial progenitors and determination of the latency of the proliferative response. J. Neurosci. 16:5466-5477, 1996.

Whitlon DS, Zhang X, Pecelunas K, Greiner MA. A temporospatial map of adhesion molecules in the organ of Corti if the mouse cochlea. J. Neurocytol. 28:955-968, 1999.

ZHENG JL, GAO WQ. Analysis of rat vestibular hair cell development and regeneration using calretinin as an early marker. J. Neurosci. 8:270-8282, 1997. 